\title{
Next Generation Diagnostics for Rare Neurological Diseases: The Future is Here
}

Can J Neurol Sci. 2014; 41: 299-300

Rare diseases, the majority of which are genetic, collectively affect as many as 1 in 12 Canadian families. ${ }^{1}$ There are an estimated 7000 rare genetic diseases, for which the underlying genetic basis is currently known for about half. ${ }^{2}$ A disproportionate number of these rare diseases affect the nervous system; resulting in a number of common phenotypes familiar to neurologists including intellectual disability, autism, epilepsy, muscular dystrophy, neuropathy and ataxia. For patients and families affected by such diseases, arriving at an underlying diagnosis (ideally confirmed by laboratory testing) is essential for enabling optimal patient management, screening, treatment, genetic counseling and access to supports in the health care and educational systems as well as society in general. Nevertheless, given that the majority of these conditions are highly genetically heterogeneous with each single disease being potentially quite rare; approximately $50 \%$ of all patients are never given a molecular diagnosis. Many of those who do receive such a diagnosis do so only after years of wait, medical tests, appointments and lack of clarity.

In this context, van Karnebeek et $a l^{3}$ report their experience with a multidisciplinary clinic for the diagnostic evaluation of children with syndromic forms of intellectual disability. Despite assessing patients that had already been reviewed by multiple services for a diagnostic opinion, they were able to provide a confirmed diagnosis in 11/24 patients (46\%) and a leading working diagnosis in an additional nine children (38\%) for a total diagnostic yield as high as $84 \%$. This dramatic diagnostic rate speaks to the success of bringing together experts from multiple services (in this instance primarily medical genetics, biochemical genetics and pediatric neurology) with overlapping but distinct expertise in the evaluation of these patients; but also to several important new technologies. The first is array comparative genomic hybridization (array CGH), a molecular cytogenetic technology which is now the recommended front line investigation for children with a suspected genetic cause of intellectual disability in Canada. ${ }^{4}$ While all of their patients had array $\mathrm{CGH}$ prior to being enrolled in the clinic, the authors demonstrated that re-evaluation of previous array $\mathrm{CGH}$ data several years later provided or contributed to a new diagnosis in $3 / 24$ patients. The other is next generation sequencing (NGS), currently primarily whole exome sequencing (WES); van Karnebeek and colleagues report that WES (typically ordered by them on a research basis) was essential to lead to a confirmed, or strong working diagnosis, in about one quarter of all study patients.

Next generation sequencing has rapidly changed the landscape for research, and subsequently clinical diagnostics, in the field of rare diseases. Since the first successful discovery of a novel gene for a rare Mendelian disease in late 2009, these technologies (primarily WES) have now been applied to the identification of over 230 novel disease genes..$^{5}$ These initial research successes have quickly moved into clinical applicability, with high diagnostic yield in cohorts of patients that were previously undiagnosed with such presentations as intellectual disability ${ }^{6}$ and ataxia. $^{7}$

Despite the clear promise of such an approach, a number of issues remain to be answered prior to broad clinical availability of NGS. One is the cost of testing; although the costs of NGS are decreasing rapidly (WES is currently available in clinical diagnostic laboratories in the USA for $\sim 5000$ ). In addition, the current costs of arriving (or not) at a diagnosis are also not insignificant. For example, Shashi et $a l^{8}$ conducted a retrospective review of 500 unselected patients referred to a clinical genetics service for diagnosis. Of the 461 patients that met study criteria, $46 \%$ received a diagnosis, with $72 \%$ of these diagnoses occurring at the first visit. The cost of making a diagnosis in patients after the first visit was $\$ 25,000$ USD. van Karnebeek and colleagues document an average cost per patient in their study at $\$ 18,119.50$ prior to entry in their diagnostic clinic and an additional $\$ 6,844.14$ of spending associated with their clinic. It is therefore reasonable to predict that judicious use of WES in appropriately selected patients early in the diagnostic process may actually have a positive impact on global spending on the diagnostic workup of these patients. Even when a specific diagnosis is suspected, targeted or specific genetic testing is often expensive, and as highlighted by the authors, access to funding for such testing is unevenly available across Canada. Thus, diagnostic application of WES for such patients will provide access to diagnostic testing they would otherwise not have available to them. A second issue is that of data interpretation, to correctly identify the pathogenic alleles causing disease in a given patient. While this topic is outside the scope of this editorial, suffice it to say such assessment will require a multidisciplinary team of clinicians (including medical geneticists and other specialists with knowledge of the patient's phenotype), molecular and other laboratory geneticists, bioinformaticians and genetic counselors; not dissimilar to the multidisciplinary clinic assembled by van Karnebeek and colleagues. A final important issue is that of the 'incidental' finding that may be uncovered in the course of interrogating the entire exome, or genome, for diagnostic purposes. While healthy debate still exists as to the best approach to such findings, the American College of Medical Genetics and Genomics (ACMG) ${ }^{9}$ has taken the approach that variants in a list of 56 genes causing 
24 inherited conditions must be sought and reported. It is clear that such testing should not be undertaken without the appropriate pre, and post-test genetic counseling.

So where are we in Canada with respect to the care of patients with rare disease in early 2014? To facilitate access for the community of patients and physicians to comprehensive information on diagnosis, care and treatment of rare disease, Canada is now an active participant member in Orphanet,,$^{10}$ the leading global information portal on rare diseases. Canada has also assumed a leadership role in the International Rare Disease Research Consortium (IRDiRC); ${ }^{11}$ a global consortium with the objectives of developing diagnostic tests for nearly all rare diseases, and 200 new therapies for rare diseases by 2020 . With respect to the delivery of clinical genomic sequencing in Canada, the Canadian College of Medical Genetics (CCMG) along with researchers from the Care4Rare consortium, ${ }^{12}$ building on the success of the FORGE (Finding of Rare Disease Genes) Canada project, are studying several issues including clinical indications for NGS, cost-effectiveness, as well as best practices around the return of incidental findings. In addition, Care4Rare is developing educational programs for trainees, not only in the field of Medical Genetics but ultimately in other branches of specialty medicine in Canada such that the "next generation" of physicians is prepared for "next generation" testing.

For a given patient with an undiagnosed condition it will be unclear at the time of testing whether such a test will identify known mutations that explain the phenotype. As such, it is recognized that WES may serve as either a discovery or a diagnostic tool in the same patient. ${ }^{13}$ It is anticipated that in many patients (such as patients 9 to 11 reported by van Karnebeek et al), WES may uncover a compelling candidate gene, never before linked to human disease. While such 'discoveries' are exciting, caution is still required prior to making definitive statements on pathogenicity that may alter patient management and genetic counseling. To this end determining causality typically requires either the identification of additional patients with variants in the same gene and a similar phenotype (genetic validation) or extensive, frequently expensive and timeconsuming, functional studies. To this end, it is critical that there are global mechanisms for data sharing amongst groups using this new technology in either a primarily clinical or research setting to arrive at as many accurate diagnoses as possible for families. This will be enabled by the willing participation not only of the testing laboratories but, most importantly, the ordering clinician who has knowledge of the patient's phenotype to enter such data such that both the phenotype and genotype are discoverable. In Canada, PhenomeCentral ${ }^{14}$ will serve as one such integrated portal for sharing and searching data in a centralized repository for difficult to diagnose rare diseases, enabling discovery and collaboration while preserving patient privacy. In this sense, despite the remarkable gains made in the past few years in terms of technology, the art of 'phenotyping' remains as important as ever as it relates to the diagnosis and management of the millions of Canadians affected with rare diseases.

\author{
A. Micheil Innes \\ University of Calgary, Calgary, Alberta, Canada \\ Kym M. Boycott \\ University of Ottawa, Ottawa, Ontario, Canada
}

\section{REFERENCES}

1. Canadian Organization for Rare Disorders. Toronto; [cited 2014 Feb 20]. Available from: http://www.raredisorders.ca/.

2. McKusick VA. Mendelian inheritance in man and its online version, OMIM. Am J Hum Genet. 2007;80(4):588-604.

3. van Karnebeek C, Murphy T, Giannasi W, Thomas M, Connolly M, Stockler S. Diagnostic value of a multidisciplinary clinic for intellectual disability. Can J Neurol Sci. 2014;41(3):333-45.

4. Duncan AM, Chodirker B. Use of array genomic hybridization technology for constitutional genetic diagnosis in Canada. Paediatr Child Health. 2011;16(4):211-12.

5. Boycott KM, Dyment DA, Sawyer SL, Vanstone MR, Beaulieu CL. Identification of genes for childhood heritable diseases. Annu Rev Med. 2014;65:19-31.

6. de Ligt J, Willemsen MH, van Bon BW, et al. Diagnostic exome sequencing in persons with severe intellectual disability. N Engl J Med. 2012;367(20):1921-9.

7. Sawyer SL, Schwartzentruber J, Beaulieu CL, et al. Exome sequencing as a diagnostic tool for pediatric-onset ataxia. Hum Mutat. 2014;35(1):45-9.

8. Shashi V, McConkie-Rosell A, Rosell B, et al. The utility of the traditional medical genetics diagnostic evaluation in the context of next-generation sequencing for undiagnosed genetic disorders. Genet Med. 2013. [EPub ahead of print Aug 8].

9. Green RC, Berg JS, Grody WW, et al. ACMG recommendations for reporting of incidental findings in clinical exome and genome sequencing. Genet Med. 2013;15:565-74.

10. Orphanet Canada. Montreal; [cited 2014 Feb 20]. Available from: http://www.orpha.net/national/CA-EN/index/homepage/.

11. International Rare Disease Research Consortium. Paris; [cited 2014 Feb 20]. Available from: http://www.irdirc.org/.

12. Care for Rare Canada. Ottawa; [cited $2014 \mathrm{Feb} 20$ ]. Available from: http://www.care4rare.ca.

13. Ku CS, Cooper DN, Polychronakos C, Naidoo N, Wu M, Soong R. Exome sequencing: dual role as a discovery and diagnostic tool. Ann Neurol. 2012;71(1):5-14.

14. PhenomeCentral. Toronto; [cited 2014 Feb 20]. Available from: https://phenomecentral.org. 